\title{
Establishment of Annular Dark-Field Scanning Confocal Electron Microscopy using a Double Aberration-Corrected Microscope
}

\author{
A. Hashimoto, ${ }^{*}$ P. Wang, ${ }^{* *}$ M. Shimojo, ${ }^{* * *, * * * * * * * * *}$ K. Mitsuishi, ${ }^{* * *, * * * * *}$ A. I. Kirkland, ${ }^{* *}$ P. D. Nellist, ${ }^{* *}$ \\ and M. Takeguchi** \\ * International Center for Young Scientists, National Institute for Materials Science, 1-2-1 Sengen, \\ Tsukuba 305-0047, Japan. \\ ** Department of Materials, University of Oxford, Parks Road, Oxford OX1 3PH, UK. \\ *** High Voltage Electron Microscopy Station, National Institute for Materials Science, 3-13 \\ Sakura, Tsukuba 305-0003, Japan \\ **** Advanced Science Research Laboratory, Saitama Institute of Technology, 1690 Fusaiji, Fukaya \\ 369-0293, Japan \\ ***** Quantum Dot Research Center, National Institute for Materials Science, 3-13 Sakura, \\ Tsukuba 305-0003, Japan \\ ****** Advanced Nano-characterization Center, National Institute for Materials Science, 3-13 \\ Sakura, Tsukuba 305-0003, Japan
}

Three-dimensional (3D) imaging and analysis with high resolution are often required in material science and engineering. Scanning confocal electron microscopy (SCEM) is one of promising 3D imaging techniques with TEM [1-3], along with computerized tomography and aberration-corrected STEM. SCEM is an analogue of scanning confocal optical microscopy, which has been demonstrated to provide sharp images of thick samples. We have developed a stage-scanning system, by which only the specimen moves three-dimensionally while maintaining constant lens configuration, and established a stage-scanning-type SCEM using a conventional TEM/STEM instrument [3]. In principle, SCEM enables us to perform optical depth sectioning by rejecting most of electrons from an out-of-focal plane in an object using a pinhole aperture at a conjugate plane, as shown in Fig.1. However, features elongated in the $\mathrm{Z}$ direction are seen in bright-field (BF) SCEM that makes use of the direct beams. This elongation arises because the object is laterally extended. Recently, we proposed annular dark-field (ADF) SCEM that use scattered electrons [4]. We additionally installed an annular aperture to block direct beams and select only scattered electrons, and finally succeeded in 3D imaging of carbon nanostructures and catalytic nanoparticles on support materials by ADF-SCEM [4,5]. Further, it was found that the depth resolution of ADF-SCEM depends on the probe size in the depth direction. Therefore, the objects were apparently elongated with about $100 \mathrm{~nm}$ along the optical axis. In this study, we established an ADF-SCEM trajectory using a double aberration-corrected electron microscope, which can provide a finer probe.

Figure 1 shows a schematic diagram of an ADF-SCEM setup with two aberration corretors and a stage-scanning system. The Oxford-JEOL 2200MCO fitted with two spherical aberration correctors was used for the present experiment. Nellist et al. have already demonstrated that confocal imaging trajectries can be established in the double aberration-corrected microscope [2]. Then, we installed the designed pinhole and annular apertures to the microscope. Because the stage-scanning system is independent from a microscope, it was easy to combine it with the aberration-corrected microscope. The upper lens forms a fine probe in a specimen and the lower lens projects and magnifies an image of the probe on a STEM detector. Detected signals are displayed on a computer screen synchronizing with the specimen displacement. 
Before observation, we simulated the typical ADF-SCEM probes that were formed by using conventional $(\mathrm{Cs}=1 \mathrm{~mm})$ and double aberration-corrected electron microscopes, as shown in Fig. 2 (a) and (b), respectively. Comparison of the two probes obviously indicates that the aberrationcorrected microscope can produce much smaller probes. Also in actual observation, the elongation of the objects along the optical axis was reduced compared with the use of the conventional instrument, as we expected. Figure 3 (a) and (b) show vertical ADF-SCEM XZ-slice images of Au nanoparticles on an amorphous carbon film taken by using the conventional and aberration-corrected instruments, respectively. The data demonstrate the further improvement of the depth resolution by the aberration correctors.

\section{References}

[1] N.J. Zaluzec, Microsc. Today 6, 8 (2003).

[2] P.D. Nellist et al., Appl. Phys. Lett. 89, 124105 (2006).

[3] M. Takeguchi et al., J. Electron Microsc. 57, 123 (2008).

[4] A. Hashimoto et al., J. Appl. Phys. 106, 086101 (2009).

[5] A. Hashimoto et al., Microsc. Microanal. (in press).

[6] A part of this research was supported by Grant-in-Aid for Scientific Research and Bilateral Joint Research Program of JSPS (Japan), the Leverhulme Trust (UK), and the EPSRC (UK).

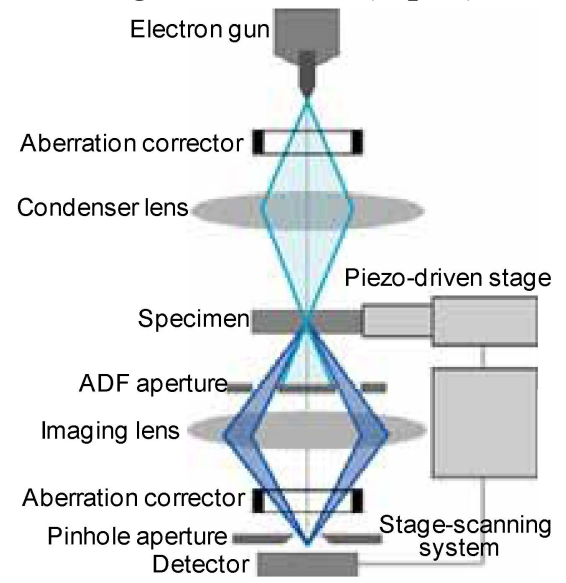

FIG.1 Schematic drawing of an ADFSCEM setup with two aberration correctors and a stage-scanning system.

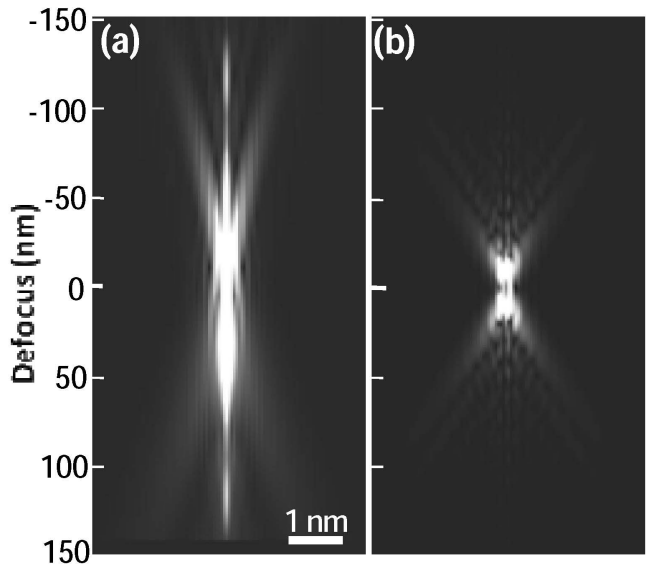

FIG.2 ADF-SCEM probes formed by using (a) conventional and (b) double aberrationcorrected microscopes.
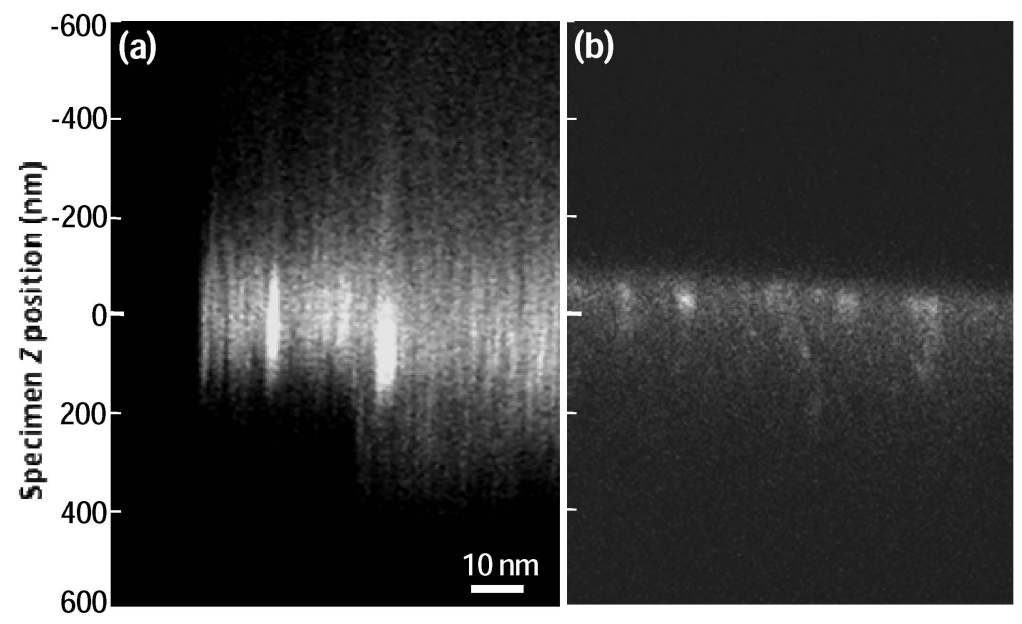

FIG.3 ADF-SCEM XZ-slice images of $\mathrm{Au}$ nanoparticles on a carbon film taken by using (a) conventional and (b) aberrationcorrected instruments. 